\title{
Design and Implementation of Hierarchical Structure-based LTE Network Application Simulation
}

\author{
Shuai Wang ${ }^{1, ~ a, ~ L i y u n ~ C h e n ~}{ }^{1, b}$ and Yongli $\mathrm{Yu}^{2, c}$ \\ ${ }^{1}$ Information Engineering Department, Ordnance Engineering College, Shijiazhuang 050003, \\ China; \\ ²Equipment Command and Management Department, Ordnance Engineering College, \\ Shijiazhuang 050003, China. \\ ashuaishuai1507@163.com, b723112756@qq.com, c1175118316@qq.com
}

Keywords: hierarchy, discrete events, LTE, simulation.

\begin{abstract}
This paper, by taking the LTE communication network as the object of study and applying the discrete event simulation method and hierarchical modeling mechanism, analyzes the LTE protocol stack and classifies it at the simulation layer; designs the functions and parameters at each simulation layer following the "protocol-equipment-platform-network" modeling process and establishes UE and eNodeB node simulation models respectively; constructs the communication network simulation scenarios of LTE multi-node accession; and gets simulation data. Experiment shows that such simulation method is of high efficiency and produces reliable simulation data.
\end{abstract}

\section{Introduction}

As the Internet and mobile communication technology develop rapidly, people's demand for mobile wireless internet access keeps growing. LTE (The Long-Term Evolution), as the extension and development of the 3G Universal Mobile Telecommunications System (UMTS), is featured by a high spectrum efficiency, a high speed of communication, a simple network structure, and smooth evolution of the existing mobile communication system, so it is the standard scheme of $4 \mathrm{G}$ cellular telecommunication access network, and the technology of choice of mobile internet access as well. It is applied extensively at present.

Based on an analysis of the LTE network, this paper studies the LTE protocol stack, and conducts classification and modeling of its structure at the simulation layer. Under the precondition of taking into full consideration the LTE frequency division duplexing (FDD) model, types of heterogeneous eNodeB antenna and other characteristics, it establishes eNodeB and UE node models based on the MAC-layer up-and-down data transmission and resource dispatching method; and build an LTE network application simulation scenario before getting simulation data based on real channel model[1][2].

\section{LTE Network}

In the LTE network system, the corresponding wireless network and core network are known as E-UTRAN (Evolved Universal Terrestrial Radio Access Network) and EPC (Evolved Packet Core), and the whole network system is named EPS (Evolved Packet System). Compared with 3G UMTS (Universal Mobile Telecommunications System), the air interface protocol, wireless transmission technology and system structure of the LTE network have qualitative changes.

In E-UTRAN, between eNodeBs at the base layer, IP transmission is applied, and transmission is logically completed through X2 interface. The eNodeBs are connected to MME/S-GW through S1 interfaces which are interlinked like a Mesh network. One eNodeB can be connected to a number of MME/S-GW, vice versa. Such design of network structure effectively supports the mobile connectivity of UE to the whole network and ensures seamless user switches.

E-UTRAN system air interface protocol stack can be classified into user-plane protocol stack and control-plane protocol stack, of which the former is similar to the UMTS system and is mainly 
composed of four layers-PHY, MAC, RLC and PDCP, and these sub-layers all end at eNodeB entities at UTRAN side. Control-plane protocol stack mainly includes NAS, RRC, PDCP, RLC, MAC and PHY layers, of which NAS ends at MME and mainly functions to realize EPS bearing management, authentication, mobility treatment in idle state, paging messages and safety control among others; RRC layer protocol ends at eNodeB and mainly provides such functions as broadcasting, paging, RRC connection management, radio bearing (RB) control, mobility management, and UE measurement reporting and control; PDCP layer provides encryption and completeness protection function; and the control plane executes the same functions as the user plane at RLC and MAC layers. Fig. 1 describes the interaction process of user-plane protocol stack, controlplane protocol stack, and different layers [3].

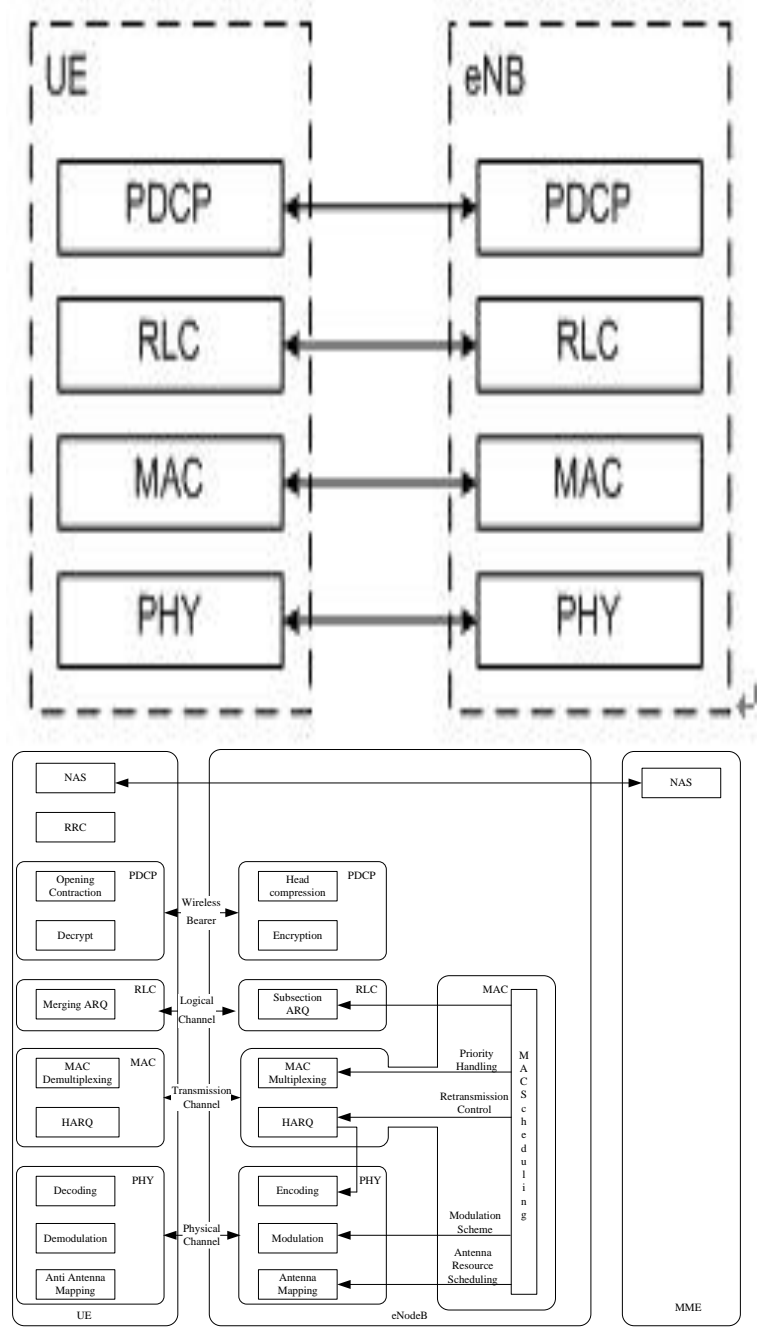

Fig. 1 LTE Protocol Stack Correspondence Relations and Interaction Flow Fig.

\section{Realization principle of LTE Network Simulation}

\subsection{Simulation Principle of Discrete Event Dynamic System.}

Discrete event dynamic system refers to an event-driven dynamic system of jumpy changes, where system switches happen at a string of discrete events, and events usually occur randomly and have complicated change relations, thus are difficult to be described with a regular equation model. The telecommunication network system is a typical discrete event dynamic system. For example, length of the router queue, and status of the communication links (busy or idle) can all be viewed as status parameters of the network simulation system.

Discrete event simulation needs to maintain a "Future Event list" (FEL), which stores all discrete events that are to happen. All these discrete events are processed by the computer one by one in strict accordance to timestamps (that is, times when discrete events occur) to ensure a strict casual relationship. That is to say, events that occur later can't be processed first. With each discrete event 
handled, the status of the simulation system will skip correspondingly. Besides, new discrete events may occur, and be inserted into the FEL for processing.

When no events are left, or it is unnecessary to continue the simulation because the model event or CPU event has reached a certain level, or statistics have become substantially accurate, simulation will end. See Fig. 2 for its working model.

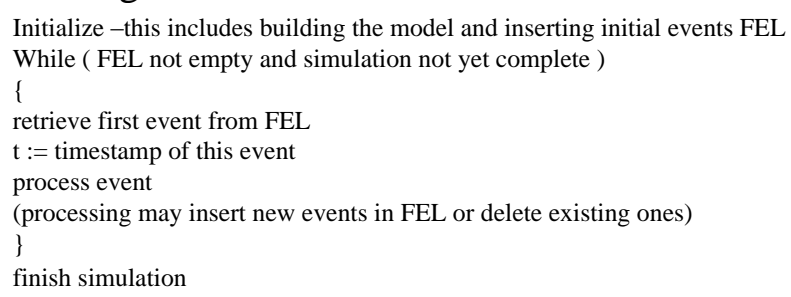

\subsection{Hierarchy Modeling Mechanism.}

Fig. 2 Discrete Event Simulations Processing

Network simulation is a special one in computer simulation. Since the transmission characteristic of network communication is electromagnetic wave, and the network input and output terminals are all application data, it can't be described by a mathematic equation. Therefore, the construction of a network simulation system is to describe the characteristics of transmission media, bits flow coding and decoding, data message sealing, convey and analysis using computer language and on the basis of analyzing, understanding and modeling network protocols. In this paper, to save the development workload to the maximum degree, code refactoring is increased, modular ideas are utilized, hierarchy modeling mechanism is applied, and the "protocol-equipment-platform-network" four-layer modeling principle is followed to build an LTE telecommunications network simulation scenario.

The protocol layer consists of all protocols and interface parameters necessary for network simulation, like TCP/IP, RIP and PPP. All these protocols are at different layers from the service perspective, like the physical layer protocol, link layer protocol, transmission layer protocol, but they have equal status from the perspective of simulation and form a big protocol assembly. Communications of protocols are realized through interfaces, and protocol combinations at different layers form an equipment simulation model.

The equipment layer consists of all equipment needed for network simulation, like switches/routers, forces stations, data link terminals. The equipment layer is a unique system structure and normally consists of physical layer, link layer, network layer, transmission layer and application layer, and integrates the protocols at physical layer, link layer, network layer, transmission layer and application layer, which can be used separately or in combination.

The platform layer refers to different platforms at network simulation like air-borne layer, satelliteborne layer and vehicle-borne layer, and is normally composed of different equipment. At network simulation, different platforms, besides having the telecommunications function, are granted such attributes as motion model, and address distribution model to control the mobile attribute of control node platform and IP distribution of internal subnets of the platforms, respectively, so as to ensure the correctness of simulation.

The network layer is the final layer of network simulation, and consists of a great number of simulation elements like links, all kinds of equipment and different platforms. The combination of such simulation elements realizes virtual simulation of a specific network. The network simulation system under the hierarchy mechanism is as shown in Fig. 1 below. 


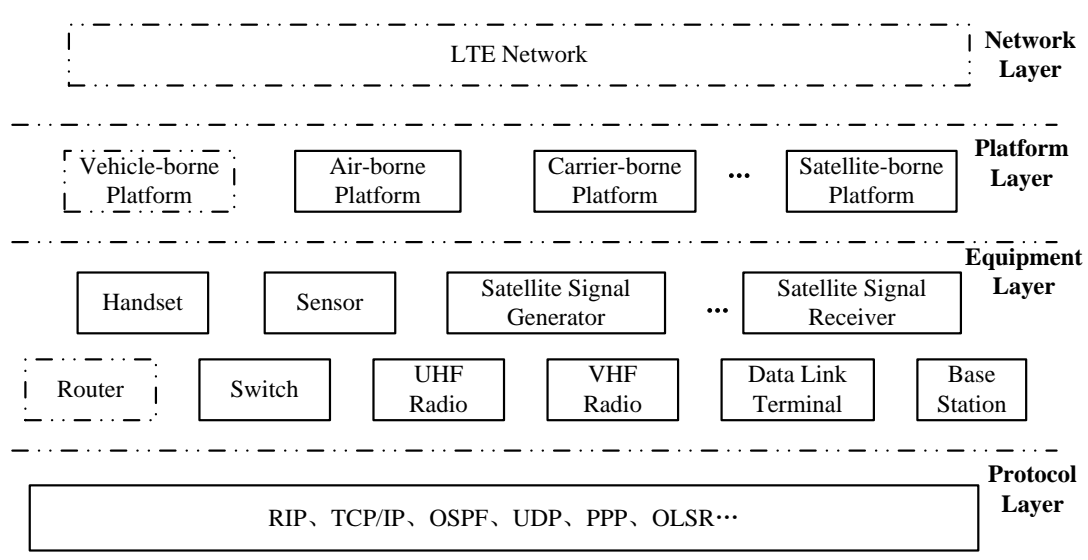

Fig. 1 Structure of the Network Simulation System under the Hierarchy Mechanism

\section{LTE network simulation Layer Design and Modeling}

This paper, by analyzing the LTE protocol stack, redesigns the NIC (Network Interface Card) system structure of LTE. LTE-NIC specifically includes the PDCP-RRC layer model, RLC layer model, MAC layer model and physical layer model. The structure of the LTE system is as shown in Fig. 4 below.

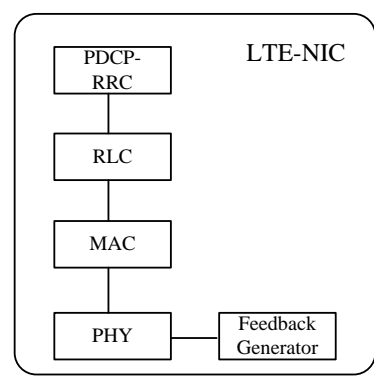

Fig. 4 NIC Module Design in UE Model

\subsection{PDCP-RRC Layer Model and Parameters.}

PDCP-RRC layer module has the functions of PDCP and RRC, of which PDCP is used for realizing such functions as IP data header compression and decompression, linking of eNodeB simulation terminals, and storage of MacNodeID; and RRC is for realizing the function of RRC linking, including the binding of local IP addresses to model ID (MacNodeId), and the distribution of locale identifiers (LCID) according to PDCP linking request. In this simulation, the (MacNodeId, LogicalCID) parameter group is used as the sole connection identifier of the whole network. PDCPRRC layer simulation realization is designed as LtePdcpRrcBase module which derives LtePdcpRrcUe, LtePdcpRrcEnb, LtePdcpRrcRelayUe and LtePdcpRrcRelayEnb simulation modules $^{[4]}$. See Table 1 for specific simulation parameters.

Table 1. PDCP-RRC Layer Model Simulation Parameters

\begin{tabular}{ccccc}
\hline Name & Styles & Default & $\begin{array}{c}\text { Value } \\
\text { Range }\end{array}$ & Description \\
\hline headerCompressedSize & int & $-1 \mathrm{~B}$ & & $\begin{array}{c}\text { Header compressed size(bytes), - } \\
\text { 1B=compression disabled }\end{array}$ \\
conversationRlc & int & 1 & $\geqq 0$ & \\
streamingRlc & int & 1 & $\geqq 0$ & \\
interactiveRlc & int & 1 & $\geqq 0$ & \\
backgroundRlc & int & 1 & $\geqq 0$ & $\begin{array}{c}\text { Statistic recording:end2end delay and } \\
\text { throughout at the mac layer }\end{array}$ \\
\hline
\end{tabular}

\subsection{RLC layer Model and Parameters.}

RLC layer is between PDCP and MAC layers, and communicates with the PDCP layer through SAP (Service Access Point) and with the MAC layer through logic channels. Data that are received by RLC module from the PDCP layer, or are sent to the PDCP layer are called RLC SDU; and data 
that are received by RLC module from the MAC layer, or are sent to the MAC layer are called RLC PDU. RLC layer simulation realization is designed as LteRlcMux module, and main functions include sectioning/cascading and reorganizing RLC SDU (applicable to the UM and AM models), and the size of SDU is designated by the MAC RLC layer; correcting errors through ARP (applicable to the AM model); re-sequencing RLC PDC (applicable to the UM and AM models); checking the repetition package (applicable to the UM and AM models); and re-segmenting RLC PDU (applicable to the AM model). See Table 2 for an explanation of the LteRlcMux parameters.

Table 2. RLC Layer Simulation Model Parameters

\begin{tabular}{ccccc}
\hline name & styles & default & value range & description \\
\hline fragmentSize & int & $30 \mathrm{~B}$ & & Fragment size (Unit: B) \\
timeout & double & $1 \mathrm{~s}$ & & RX Buffer timeout \\
maxRtx & int & 3 & $\geqq 0$ & \\
pduRtxTimeout & double & $2.0 \mathrm{~s}$ & $\geqq 0$ & \\
ctrlpduRtxTimeout & double & $2.0 \mathrm{~s}$ & $\geqq 0$ & \\
bufferStatusTimeout & double & $2.0 \mathrm{~s}$ & $\geqq 0$ & \\
txWindowSize & int & 200 & $\geqq 0$ & \\
\hline
\end{tabular}

\subsection{MAC layer model and parameters.}

LET MAC layer module realizes effective linking between RLC layer business and physical layer, and its main functions include mapping between logic and transmission channels; choice of the transmission format; error correction through the HARQ mechanism; and RLC PDU reuse and dereuse. LTE MAC layer simulation realization is designed as LteMacBase module which derives LteMacUe, LteMacEnb, LteMacRelayUe and LteMacRelayEnb models. See Table 3 for an explanation of relevant parameters.

Table 3. MAC Layer Simulation Model Parameters

\begin{tabular}{ccccc}
\hline Name & Styles & Default & Value Range & Description \\
\hline queueSize & int & 2MB & & MAC Buffer queue size \\
maxBytesPerTti & int & $3000 \mathrm{~KB}$ & & MAC MIB \\
muMimo & bool & true & True/false & \\
harqProcesses & int & 8 & $\geqq 0$ & \\
maxHarqRtx & int & 4 & $\geqq 0$ & \\
\hline
\end{tabular}

\subsection{Physical layer model and parameters.}

The physical layer module provides data transmission service for higher levels by simulating transmission channels, and is mainly used to calculate the SINR of receiving nodes, calculating CQIs, and estimating the channel transmission error rate. The LTE PHY layer simulation realization is designed as LtePhyBase module and derives such models as LtePhyUe, LtePhyEnb, LtePhyRelayUe and LtePhyRelayEnb. See Table 4 for an explanation of relevant parameters. Relevant module that is used in cooperation with the physical layer also includes the ChannelModel Class.

Table 4. Physical Layer Model Parameters

\begin{tabular}{|c|c|c|c|c|}
\hline Name & Styles & Default & Value Range & Description \\
\hline usePropagationDelay & bool & true & true/false & $\begin{array}{c}\text { Should transmission delay be } \\
\text { simulated? }\end{array}$ \\
\hline channelModel & xml & & & \\
\hline ueTxPower & double & 26 & & \\
\hline eNodeBTxPower & double & 46 & & \\
\hline relayTxPower & double & 20 & & \\
\hline microTxPower & double & 30 & & \\
\hline txDirection & String & "OMNI" & & \\
\hline txAngle & int & 0 & $\geqq 0$ & $\begin{array}{c}\text { \#CQI } \\
\text { statics@statistic[averageCqi] }\end{array}$ \\
\hline
\end{tabular}




\subsection{UE and eNodeB node simulation models.}

To realize LTE telecommunication network simulation, it is also necessary to build a UE node model and an eNodeB model. According to the aforesaid classification, UE simulation model can be divided into application layer, transmission layer, network layer and LTE-NIC layer, and its main functions include simulating telecommunication terminals, namely initiating mobile phone registration and base station linking; supporting voice calls and data businesses, etc. eNodeB simulation model can be classified into application layer, transmission layer, network layer, and LTENIC layer, while being compatible with the interface module of IP core network, and its main functions include: simulating base station functions, completing resource dispatching, managing wireless resources, and realizing UE equipment wireless access and mobility management. See Fig. 5 for the structures of UE simulation model and eNodeB simulation model.
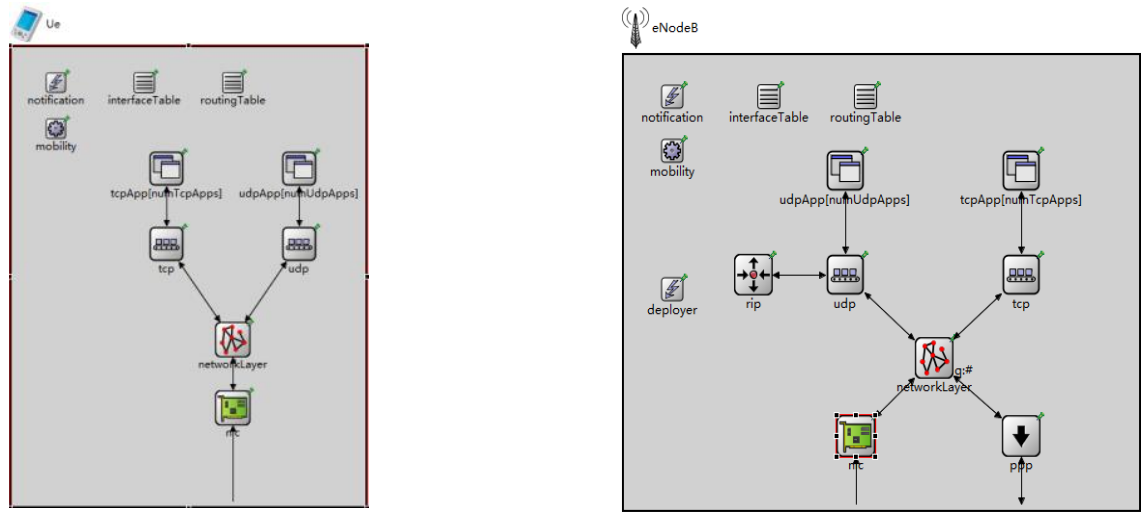

Fig. 5 Simulation UE Node Model and eNodeB Node Model

\section{Realization of LTE network application simulation}

What is realized in this network simulation is a multi-cell telecommunications scenario. According to the aforementioned eNodeB model and UE model, it designs two eNodeBs and four UEs in the simulation scenario. In the simulation process, the VoIP congestion model is applied, and UE model is linked to some server of the Internet and communicates with the latter; the server defines application layer procedures corresponding to UE numbers, and the network layer configuration is IPv4 protocol to ensure reasonable distribution of UE models that are linked to different eNodeB1 and eNodeB2 base stations at different network sections. Parameters "macCellId" and "masterId" used are interconnected through $\mathrm{eNodeB}$, and UE and eNodeB were designated locations and mobile parameters. The total length of simulation was 600 seconds, single-step simulation, network bandwidth specified as $80 \mathrm{Mb} / \mathrm{s}$, network load is set to 1024 . End-to-end delay, IP forwarding throughout, IP receiving throughout were selected as the performance indicators of LTE network evaluation. See Fig. 6 for simulation operation of LTE network, and see Fig. 7 for statistics of vector data of the simulation results. Refer to Table 5 for main data statistical results[5].

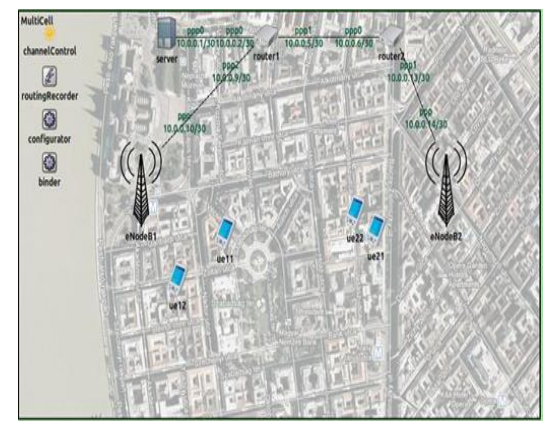

Figure 6 Telecommunications Network Operation Effect 


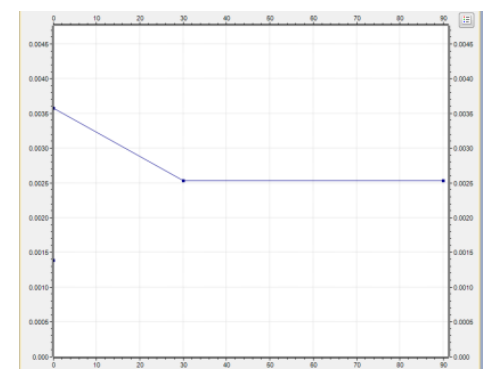

a End-to-end delay (Unit: $\mathrm{ms}$ )

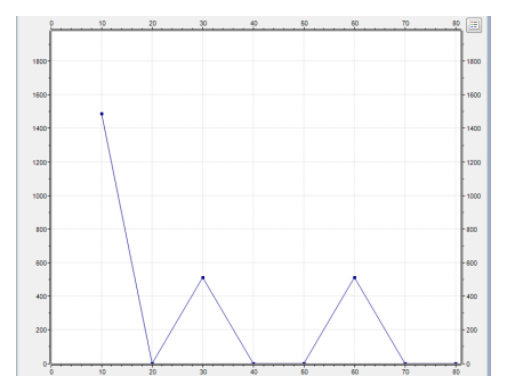

b Forwarding throughout (Bit/s)

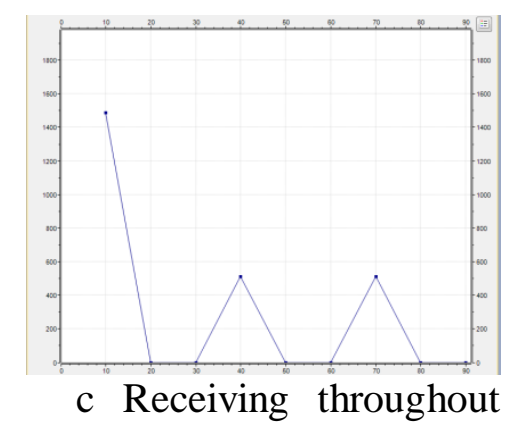
$(\mathrm{Bit} / \mathrm{s})$

Fig. 7 LTE Network Simulation Data

Table 5 Statistics on Main LTE Network Simulation Performance Indicators

\begin{tabular}{ccccc}
\hline & $\begin{array}{c}\text { End-to-end } \\
\text { delay } \\
\text { (unit: } \mathrm{ms})\end{array}$ & $\begin{array}{c}\text { IP sending } \\
\text { throughout } \\
(\text { Bit/s) }\end{array}$ & $\begin{array}{c}\text { IP receiving } \\
\text { throughout(Bit/s) }\end{array}$ & $\begin{array}{c}\text { IP forwarding } \\
\text { throughout(Bit/s) }\end{array}$ \\
\hline MultiCell & 0.02511 & 238010.18 & 224857.89 & 238010.18 \\
MultiCell.router1 & 0.01994 & 15307.88 & 293.33 & 1537.88 \\
MultiCell.router2 & 0.02509 & 769.88 & 435.56 & 769.88 \\
\hline
\end{tabular}

\section{Conclusion}

After the operation of the above LTE simulation network ended, we can see that the average endto-end delay of the whole network was about $0.025 \mathrm{~ms}$, the average value of IP forwarding throughout was $238010 \mathrm{Bit} / \mathrm{s}$, and the average value of IP receiving throughout was 224857Bit/s. Experiment shows that applying the hierarchy modeling idea and modular structure and being driven by discrete events is an effective way of studying LTE network telecommunication simulation; and that the simulation process is highly real, and the simulation data are reliable.

\section{References}

[1]. WANG Zhen-hao, HU Ya-hui, CI Song. QoE-oriented HIL Simulation Platform for Mobile Streaming in LTE System[J]. Journal of System Simulation, Vol.26 No.3. Mar.2014:556-561.

[2]. HE Jinwei, DING Lu, LIU Zhimin. Realization and optimization of LTE system-layer simulation platform[J]. Computer Engineering and Application,2012, 48(22):104-108.

[3]. XU Bing, XIE Zhi-jun. Research and Technologies of LTE System-layer Simulation [J].Radio Communications Technology. Vol.40 No.5 2014:9-12.

[4]. [Yang Qun. The Radio Access Network Planning and Simulation of LTE Networks[D].Nanjing: Nanjing University of Posts.2015.

[5]. WANG Xiaofeng. Study on the Methods to Improve the Performace of Large-Scale Discrete Event Network Simulation[D]. Harbin: Harbin Institute of Technology.2007. 